WATER PHASES

\section{Experimental observation of the liquid-liquid transition in bulk supercooled water under pressure}

\author{
Kyung Hwan Kim ${ }^{1,2 *}$, Katrin Amann-Winkel ${ }^{1 *}$, Nicolas Giovambattista ${ }^{3,4}$, Alexander Späh ${ }^{1}$, \\ Fivos Perakis ${ }^{1}$, Harshad Pathak ${ }^{1}$, Marjorie Ladd Parada ${ }^{1}$, Cheolhee Yang ${ }^{2}$, Daniel Mariedahl ${ }^{1}$, \\ Tobias Eklund ${ }^{1}$, Thomas. J. Lane ${ }^{5,6}$, Seonju You ${ }^{2}$, Sangmin Jeong ${ }^{2}$, Matthew Weston ${ }^{1}$, Jae Hyuk Lee, \\ Intae Eom? ${ }^{7}$ Minseok Kim? , Jaeku Park7, Sae Hwan Chun ${ }^{7}$, Peter H. Poole ${ }^{8}$, Anders Nilsson ${ }^{1} \dagger$
}

We prepared bulk samples of supercooled liquid water under pressure by isochoric heating of highdensity amorphous ice to temperatures of $205 \pm 10$ kelvin, using an infrared femtosecond laser. Because the sample density is preserved during the ultrafast heating, we could estimate an initial internal pressure of 2.5 to 3.5 kilobar in the high-density liquid phase. After heating, the sample expanded rapidly, and we captured the resulting decompression process with femtosecond $x$-ray laser pulses at different pump-probe delay times. A discontinuous structural change occurred in which low-density liquid domains appeared and grew on time scales between 20 nanoseconds to 3 microseconds, whereas crystallization occurs on time scales of 3 to 50 microseconds. The dynamics of the two processes being separated by more than one order of magnitude provides support for a liquid-liquid transition in bulk supercooled water.

T he discovery of the apparent divergence of isothermal compressibility $(I)$ and heat capacity $\left(C_{\mathrm{P}}\right)(2)$ as water is supercooled has inspired many theoretical scenarios to explain the origin of this anomalous behavior (3-5). One popular hypothesis proposes the existence of a liquid-liquid transition (LLT) in supercooled water between highdensity liquid (HDL) and low-density liquid (LDL), terminating at a liquid-liquid critical point (LLCP) at positive pressures $(3,6)$. The anomalous behavior of water in this hypothesis is attributed to fluctuations emanating from the LLCP. Recently, the structure of supercooled water was found to change continuously upon cooling at 1 bar down to $227 \mathrm{~K}(7,8)$, indicating one-phase behavior without an LLT at ambient pressure. Therefore, the experimental results imply that if the LLT indeed exists, the associated LLCP must be located at pressure $(P)>1$ bar (3). Rapid ice formation in conditions at which the LLT has been proposed has restricted studies of pure bulk water to computer simulations, with some models exhibiting an LLT and others not (6,9-16). Although measurements on amorphous ice

\footnotetext{
Department of Physics, AlbaNova University Center, Stockholm University, SE-10691 Stockholm, Sweden. 2Department of Chemistry, Pohang University of Science and Technology (POSTECH), Pohang 37673, Republic of Korea. ${ }^{3}$ Department of Physics, Brooklyn College of the City University of New York, Brooklyn, NY 11210, USA. ${ }^{4}$ Ph.D. Programs in Chemistry and Physics. The Graduate Center of the City University of New York, New York, NY 10016. USA

${ }^{5}$ SLAC National Accelerator Laboratory, 2575 Sand Hill Road, Menlo Park, CA 94025, USA. ${ }^{6}$ Deutsches ElektronenSynchrotron (DESY), Notkestrasse 85, 22607 Hamburg, Germany. ${ }^{7}$ Pohang Accelerator Laboratory, Pohang, Gyeongbuk 37673, Republic of Korea. ${ }^{8}$ Department of Physics, St. Francis Xavier University, Antigonish, NS B2G

2W5, Canada.
*These authors equally contributed to this work.

†Corresponding author. Email: andersn@fysik.su.se
}

and ultraviscous supercooled water at temperatures in the range of 115 to $150 \mathrm{~K}$ (17-19) are consistent with a LLT, it has been argued that these observations are not related to real liquid states (20-22) and cannot be directly connected to the two proposed liquid phases at higher temperatures.

An LLT has previously been detected in phosphorus, where the structure factor in x-ray scattering showed a discontinuous change with varying pressure (23). Also, in interfacial ice, a nonequilibrium phase transition was observed as a discontinuous change and coexistence of peaks in diffraction experiments (24). On the basis of neutron-scattering experiments of water, it has been proposed that distinct HDL and LDL phases may be identified by their well-defined peak positions in the structure factor (25). In particular, the position of the first peak in the $\mathrm{O}-\mathrm{O}$ scattering is strongly sensitive to the existence of tetrahedral structures (LDL) or interstitial molecules between the first and second shells (HDL) $(25-27)$. Hence, the most direct way to detect an LLT in supercooled water may be to follow the liquid structure with $\mathrm{x}$-ray or neutron scattering and observe whether the scattering peaks undergo a discontinuous change like in phosphorus and interfacial ice, but with positions as predicted from the neutronscattering results for water. The challenge is to conduct such an experiment at different pressures and on a time scale short enough so that the LLT may be observed before ice crystallization occurs.

Recent experimental approaches have allowed access to deeply supercooled water under conditions in which ice crystallization is rapid-for example, fast cooling of micrometersized droplets and ultrafast probing with an $\mathrm{x}$-ray laser $(7,8)$ as well as nanosecond heat- ing of thin ice films into the liquid state (28). However, these studies have been limited to atmospheric pressure (1 bar) rather than the high-pressure conditions of the proposed LLT. In order to avoid crystallization, and given that the presence of ions has a similar effect as that of pressure on water, another recent approach has been to use highly concentrated supercooled aqueous solutions, which gives results indicating the existence of an LLT (29). However, it is unclear whether these results can be connected to an LLT in pure bulk supercooled water under conditions in which fast ice crystallization occurs.

We used a new compression-decompression procedure carried out on ultrafast time scales, where the initial pressure increase was imposed by laser-pulse-induced heating. When the time scale of the laser-induced energy release is much shorter than the time for sound to travel through the sample [valid with heating time scales of $<0.1 \mathrm{~ns}$ for a $>0.1-\mu \mathrm{m}$-thick water film (30)], the heating is isochoric, and the pressure inside the sample increases considerably. After the ultrafast laser pulse ends, the sample expands rapidly as the internal pressure decreases toward that of the surroundings. However, if the liquid dynamics are fast enough to relax the sample on a time scale shorter than the time for expansion, quasi-equilibrium behavior will be observed during the decompression process. We probed the system with $\mathrm{x}$-ray scattering at different time delays during the decompression and observed a sudden change in the structure factor, which is indicative of a discontinuous LLT. We also detected ice crystallization occurring at longer time scales, confirming that the LLT is metastable and distinct from the liquid-ice transition.

We mounted high-density amorphous (HDA) ice samples in a cryostat inside a vacuum chamber to allow for pump-probe measurements in a transmission geometry, using infrared and x-ray lasers (Fig. 1A). Our samples were prepared ex situ under pressure and quenched-recovered at $78 \mathrm{~K}$, at which the temperature was low enough to kinetically arrest the sample. The chosen samples in the measurement had a thickness of either 35 to $55 \mu \mathrm{m}$ or 15 to $25 \mu \mathrm{m}$. They were then pumped by a $100-f s, 2-\mu \mathrm{m}$-wavelength infrared (IR) pulse, which excited a combination of $\mathrm{O}-\mathrm{H}$ stretch and $\mathrm{H}-\mathrm{O}-\mathrm{H}$ bending modes that rapidly decayed into heat (31), and increased the temperature on a time scale of $\approx 20 \mathrm{ps}(32)$. Excitations of the electron system may also be possible because our laser fluence $\left(6.5 \mathrm{~J} / \mathrm{cm}^{2}\right)$ was above the threshold known for nonlinear optical breakdown $(33,34)$. However, no noticeable higher order behavior was observed in our experimental data, indicating that there is not a prominent contribution from a nonlinear process (35). 

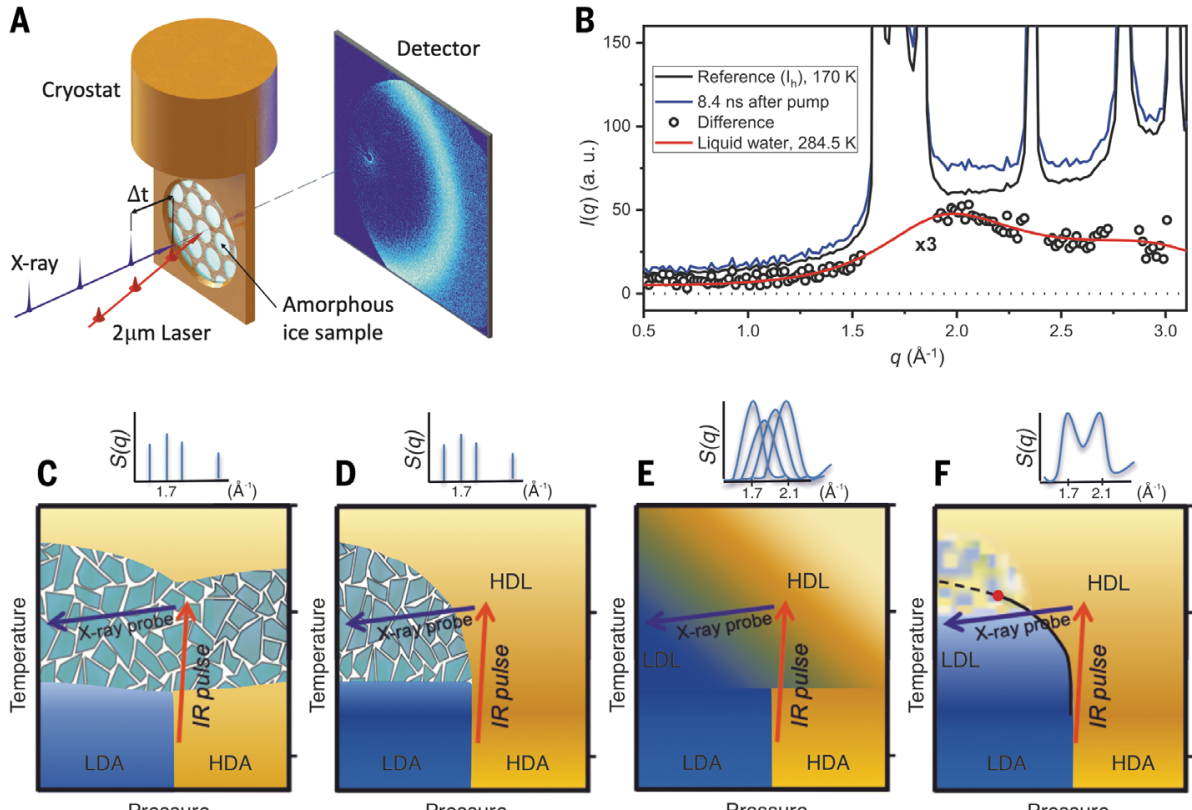

Pressure

Pressure

Pressure

Fig. 1. Experimental set-up and different possible outcomes of the experiment. (A) Schematic of the experimental setup, with a sample holder containing a Cu-grid where amorphous ice samples are mounted, pump-probe scheme with $I R$ and $x$-ray laser pulses, and a $2 \mathrm{D}$ detector for $\mathrm{x}$-ray scattering measurements. (B) Laser-induced melting of ice $\mathrm{I}_{\mathrm{h}}$ from a $170 \mathrm{~K}$ base temperature. The difference between the 8.4-ns delay and the unpumped sample omits regions where intense Bragg peaks lie. The difference data are compared with previously measured water scattering at $284.5 \mathrm{~K}$ (41). The difference is multiplied by a factor of 3 for clarity. ( $\mathbf{C}$ to $\mathbf{F}$ ) Four different hypotheses for the potential scenarios during the pump-probe experiment. At low temperatures, the equilibrium phase boundary between LDA and HDA is depicted. The original pressure of HDA is estimated from its preparation by pressure annealing and assuming constant sample density (35). The small sketches on top of the figures illustrate the evolution of the structure factor $S(q)$ probed with $x$-ray pulses along the blue arrow in the phase diagram. (C) HDA crystallizes immediately after exposure to the IR pump pulse (red arrow), resulting in Bragg-reflections in $S(q)$. (D) HDA transforms into supercooled water, followed by crystallization, resulting in Bragg peaks but at longer delay times.

(E) Transformation into supercooled water, followed by a continuous transition from HDL to LDL, during which the first diffraction maximum in $S(q)$ is expected to shift continuously to lower-momentum transfers q. (F) $S(q)$ develops a second maximum at $q=1.7 \AA^{-1}$ as LDL forms, which coexists with the maximum at $q=2.1 \AA^{-1}$ associated with HDL, which is consistent with a first-order phase transition between HDL and LDL as proposed by the LLT scenario.

A $2-\mu \mathrm{m}$ IR pulse can superheat ice and partially melt it within $10 \mathrm{~ns}(36)$, and such partial melting was achieved with the current setup for a $100-\mu \mathrm{m}$-thick hexagonal ice $\left(\mathrm{I}_{\mathrm{h}}\right)$ sample (Fig. 1B) (35). When we applied equivalent heating to our HDA samples $(<55 \mu \mathrm{m}$ thick), the temperature increased from 115 to $\sim 205 \pm 10 \mathrm{~K}$, as estimated from temperatureinduced shifts in the Bragg reflections after crystallization occurred (35). We could estimate the pressure of the sample immediately after the IR was applied by using existing experimental data for liquid water. The density of HDA is known to be in the range of 1.13 to $1.16 \pm 0.02 \mathrm{~g} / \mathrm{cm}^{3}$ (37), and isochoric heating maintained the density initially after the IR pump. From pressure-dependent measurements of density and temperature of supercooled water $(38,39)$, we derived that the pressure after the IR pulse was between 2.5 and $3.5 \mathrm{kbar}$.
After the IR pulse was applied, spontaneous decompression began, during which the temperature remained approximately constant until cooling through heat conduction became essential after $\sim 100 \mu$ s (35). This isothermal decompression carried the sample downward in pressure, and we probed the samples with intense $<50$-fs hard x-ray pulses of $9.7 \mathrm{keV}$ at various time delays (8.4 $\mathrm{ns}$ to $1 \mathrm{~ms}$ ) with respect to the IR pulse. Scattering patterns were recorded from individual $\mathrm{x}$-ray shots on a large two-dimensional (2D) detector. For each time delay, 20 images were summed together, for which each image was measured with an x-ray shot taken at a fresh sample position.

In Fig. 1, C to F, we show predictions for the structure factor $S(q)$ as a function of momentum transfer $q$ during decompression in four hypothetical scenarios: (i) Shown in Fig. 1C, direct crystallization to the high-pressure phase ice IX or $\mathrm{I}_{\mathrm{h}}$ occurred immediately after the IR pulse was applied, because these are the underlying stable crystal phases. (ii) Shown in Fig. 1D, we assumed that only a single liquid phase existed that first appeared and then directly transformed into ice $I_{h}$ or stacking disordered ice $\left(\mathrm{I}_{\mathrm{sd}}\right)$ as the liquid decompressed (10). (iii) Shown in Fig. 1E, a continuous crossover from HDL to LDL occurred during decompression before crystallization (4). (iv) Shown in Fig. 1F, there was a discontinuous LLT from HDL to LDL before crystallization (3).

In cases (i) and (ii), we expected to see Bragg peaks in $S(q)$ caused by crystallization before any indication of the LDL structure. In case (iii), the first peak of $S(q)$ would shift smoothly with $q$ upon decompression, similar to variations observed with pressure for $300 \mathrm{~K}$ water (40) or with temperature for 1 bar water $(8,41)$. Last, in case (iv), for which there would be a discontinuous LLT involving distinct macroscopic phases, the HDL peak in $S(q)$ would remain at fixed $q$, and a new peak would appear and remain fixed at a different $q$ (that of LDL) during decompression $(18,42)$, similar to the previously observed LLT in phosporous (23).

The x-ray scattering intensity of the 35- to $55-\mu \mathrm{m}$-thick HDA samples is shown in Fig. 2A at various time delays after the IR pulse. The peak position before the IR pulse was at $q=$ $2.15 \AA^{-1}$, which is consistent with recent studies of $\operatorname{HDA}(18,27)$. At $8.4 \mathrm{~ns}$ after the IR pulse was applied, the samples had undergone heating, and we observed that the peak position remained constant and near to that of liquid water at $300 \mathrm{~K}$ and pressures of 2 to $3 \mathrm{kbar}$ (40). After $16.8 \mathrm{~ns}$, a shoulder appeared at $q=$ $1.7 \AA^{-1}$-a similar $q$ position to that of LDA and LDL $(25,27)$. This peak grew in intensity as decompression continued, up to a time delay of $3 \mu \mathrm{s}$. At longer time delays ( $3 \mu \mathrm{s}$ to $1 \mathrm{~ms}$ ), we observed the development of Bragg peaks corresponding to $I_{\mathrm{sd}}$ that increased over time. At the final time delay measured (1 ms), all samples had converted into ice.

Selected time delays of thinner samples (15 to $25 \mu \mathrm{m}$ thickness) where the amount of HDL conversion to LDL was enhanced in comparison with the thicker ones are shown in Fig. $2 \mathrm{~B}$. After $1 \mu \mathrm{s}$, there is almost a 1:1 ratio of the two components. This enhancement was consistent with thinner samples having a more uniform heating than that of thicker samples, where the IR light is absorbed more at the front than at the back surface, leading to a larger temperature gradient (35). The two observed interconverting phases have $q$ positions near HDL and LDL, as previously estimated from the extrapolation of temperature- and pressure-dependent neutron-scattering data of water at higher temperatures (25).

The scenarios in Fig. 1, D to F, can be applicable only if the sample after the IR pulse 
was a liquid, rather than an amorphous solid, and remained liquid during the decompression process. Much evidence supports this interpretation. Immediately after the IR pulse, the sample was driven to a point in the phase diagram lying above the homogeneous ice nucleation temperature $\left(T_{\mathrm{H}}\right)$, conditions that are consistent with relatively fast liquid-like diffusion. In this region, water has been observed as a metastable liquid on a time scale of minutes before transforming to high-pressure crystalline ice phases (43). We observed no Bragg peaks corresponding to such crystalline ice phases at any delay times up to $1 \mathrm{~ms}$, which
A

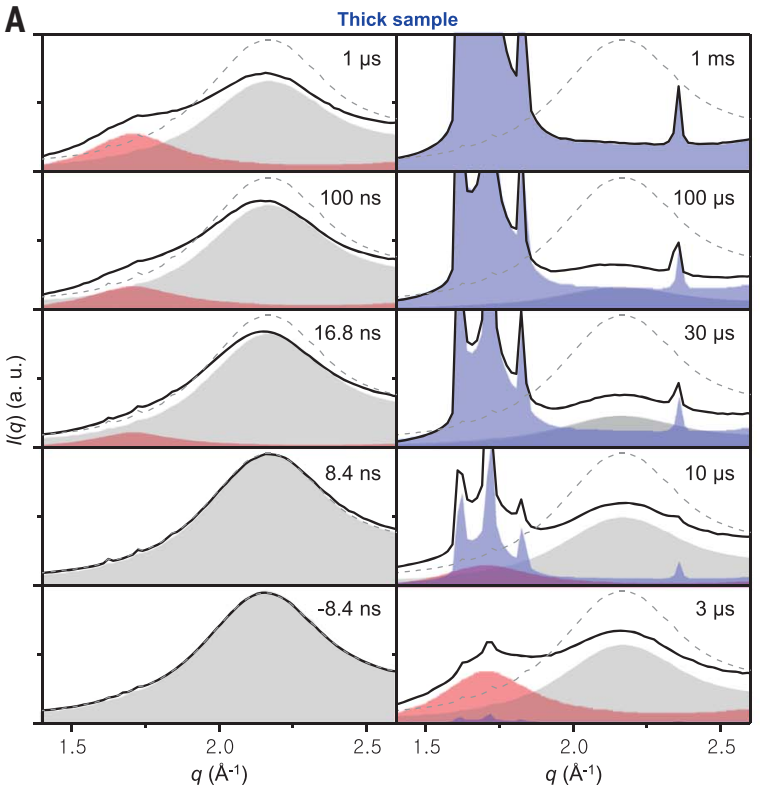

B

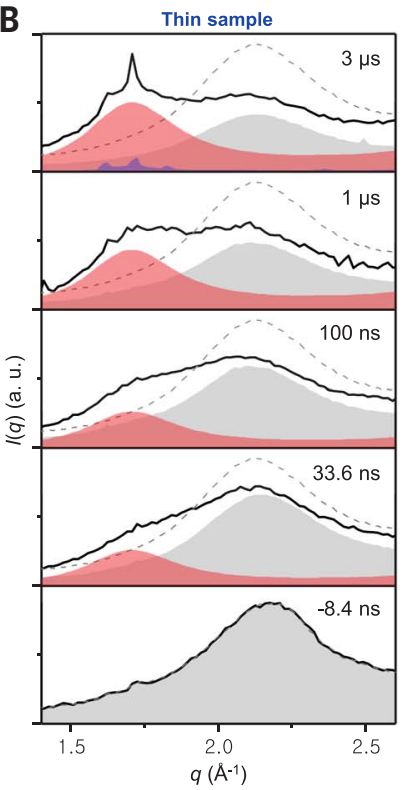

Fig. 2. Wde-angle x-ray scattering following the LLT from HDL to LDL. (A) Experimental x-ray scattering intensities, $I(q)$, of HDA samples of thickness 35 to $55 \mu \mathrm{m}$ measured before (gray dashed line) and after (black solid line) the laser excitation. Data obtained at IR pump/x-ray probe delay times of $-8.4 \mathrm{~ns}$ to $1 \mathrm{~ms}$ are shown. The contributions from HDL, LDL, and crystalline ice are indicated as gray, red, and blue shaded areas, respectively. (B) I(q) curves of thinner HDA samples (15 to $25 \mu \mathrm{m}$ thickness) after the laser excitation.

A

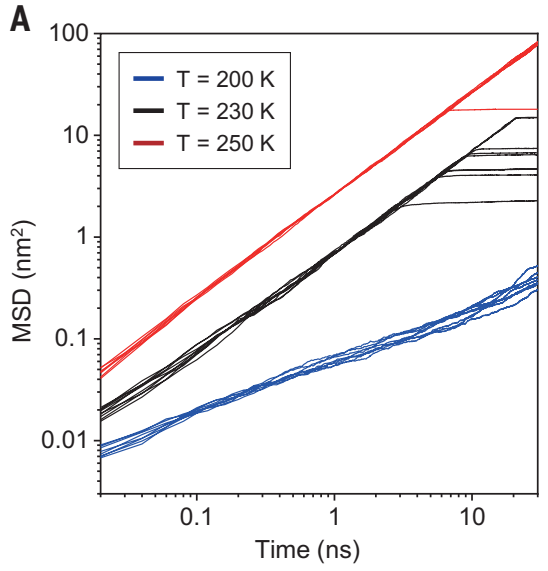

B

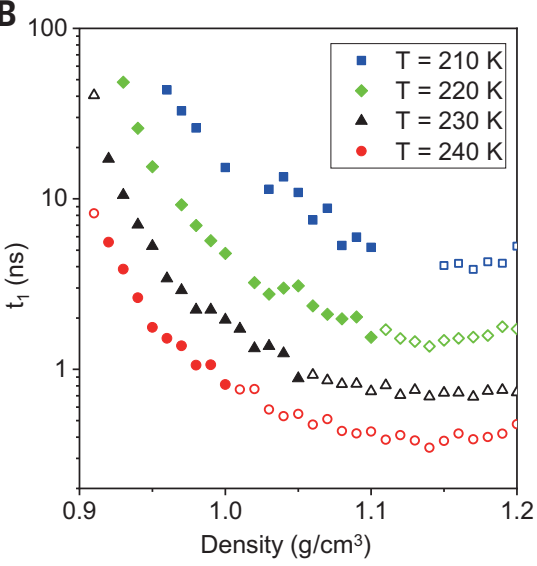

is consistent with the sample being in a metastable liquid state above $T_{\mathrm{H}}$.

To understand the immediate appearance of liquid-like diffusion after the IR-pulse heating of HDA, we used molecular dynamic simulations of the ST2 water model. There was a temperature offset of $25 \mathrm{~K}$, meaning that the experimental temperature of $205 \mathrm{~K}$ corresponds to $\sim 230 \mathrm{~K}$ in ST2 water (35). The mean-square displacement (MSD) of ST2 molecules are shown in Fig. 3A as function of time after rapid heating (at $3000 \mathrm{~K} / \mathrm{ns}$ ) of HDA. Starting at $80 \mathrm{~K}, \mathrm{HDA}$ was heated to one of three different final temperatures in the range from 200 to $250 \mathrm{~K}$ and was then held constant. If there were a delay for the sample to enter the liquid state, the MSD would be initially constant and then increase linearly after the delay.

In our simulations, we saw that the MSD immediately increased linearly with time, as expected for a diffusing liquid. From these results, we can state that within $20 \mathrm{ps}$, after fast heating from HDA, a liquid state was obtained. This process was much faster than the partial melting of ice $\mathrm{I}_{\mathrm{h}}$ by our IR pulse, which took 10 ns (Fig. 1B). However, crystal melting, a transition between phases with qualitatively different structures, is an activated process that requires crossing a free-energy barrier. Our experimental HDA samples were held for 0.5 to 5 hours at $115 \mathrm{~K}$, near the glass transition temperature of HDA $(18,37)$, so before heating, they were already in an ultraviscous liquid state. The samples encountered no freeenergy barrier on heating from 115 to $205 \mathrm{~K}$, which is consistent with HDA and HDL being structurally closely related, and as a result, the

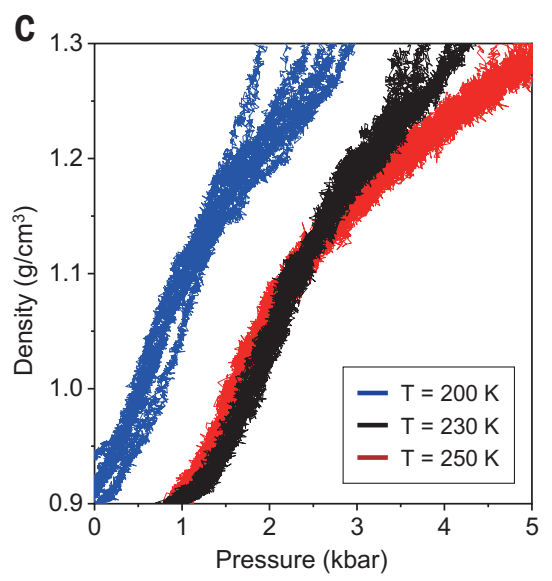

Fig. 3. Molecular dynamics simulation. (A) MSD of water molecules as a function of time during annealing of ultrafast heated HDA at $T=200$ (blue), 230 (black), and $250 \mathrm{~K}$ (red) and at density $1.30 \mathrm{~g} / \mathrm{cm}^{3}$. At $T=230 \mathrm{~K}$, all trajectories crystallize to a high-pressure ice form after $t_{x}=3$ to $20 \mathrm{~ns}$, and hence, the corresponding $\mathrm{MSD}(t)$ becomes constant at $t>t_{x}$. Some trajectories crystallize at $T=250 \mathrm{~K}$ as well, but no crystallization occurs at $T=200 \mathrm{~K}$ (within $30 \mathrm{~ns}$ ).

(B) Relaxation time, $t_{1}$, of liquid water as a function of density for several isotherms at 210 (blue), 220 (green), 230 (black), and $240 \mathrm{~K}$ (red). We computed $t_{1}$ using $t_{1}=\left(1 \mathrm{~nm}^{2}\right) /(6 D)$, where $D$ is the diffusion coefficient. Open symbols correspond to homogenous liquid systems that are either pure HDL or pure LDL, and solid symbols correspond to systems that have phase-separated into a mixture of coexisting HDL and LDL regions. (C) Density as a function of pressure during the decompression of ultrafast heated HDA at $T=200$ (blue), 230 (black), and $250 \mathrm{~K}$ (red). The decompression rate is $3 \mathrm{kbar} / \mathrm{ns}$. 
onset of fast diffusion was immediate (44), as confirmed by our simulations when heated from the amorphous state starting from either $80 \mathrm{~K}$ or $115 \mathrm{~K}$

The diffusion coefficient $D$ for liquid water at $205 \mathrm{~K}$ and $3 \mathrm{kbar}$ can be interpolated from experimental data (45), from which we obtained $D=2 \times 10^{-11} \mathrm{~m}^{2} / \mathrm{s}$. To convert $D$ to a characteristic time for liquid-like diffusion, we define $t_{1}=\left(1 \mathrm{~nm}^{2}\right) / 6 D$, where $t_{1}$ is the average time required for the MSD to reach $1 \mathrm{~nm}^{2}$, which is equivalent to diffusion of over three times the diameter of a water molecule. We found that immediately after the IR pulse, $t_{1}$ was $8 \mathrm{~ns}$. For the ST2 model, when the system had a temperature in the range from 220 to $240 \mathrm{~K}$ and a density of $1.2 \mathrm{~g} / \mathrm{cm}^{3}$, the time $t_{1}$ was between 0.5 and 2 ns (Fig. 3B), which is consistent with the time scale found experimentally and confirms the temperature offset of ST2 water. Even after our shortest delay time ( $8.4 \mathrm{~ns}$ ), the sample produced by the IR pulse had ample time to access the liquid state of HDL.

The time scale for liquid-like relaxation in the low-density regions that formed in our samples during decompression could be estimated in several ways. Previous measurements in thin layers of LDL water at 1 bar and $205 \mathrm{~K}$ found $D=2 \times 10^{-13} \mathrm{~m}^{2} / \mathrm{s}(28)$, corresponding to $t_{1}=800 \mathrm{~ns}$, a factor of about 100 times longer than the HDL formed after the IR pulse. Consistent with this factor, for ST2 water, we found that $t_{1}$ increased by a factor of $\sim 50$ as the system converted from pure HDL to pure LDL (Fig. 3B). Also, experimental crystallization times on the order of milliseconds were observed for an LDL liquid at $160 \mathrm{~K}$ obtained after fast decompression of high-pressure crystalline ice VIII (46) and could be modeled by using liquid-like diffusion. At $205 \mathrm{~K}$, we observed crystallization on a time scale of $10 \mu \mathrm{s}$, indicating an LDL state with much greater molecular mobility than at $160 \mathrm{~K}$. Furthermore, at $T>200 \mathrm{~K}$, the density varied almost instantaneously in the ST2 model during fast decompression (Fig. 3C), which is in agreement with our observation that the transformation rate in the experiment was limited only by the speed of sound in the sample (35).

Both experiments and simulations indicated that a liquid-like equilibrium was established at $205 \mathrm{~K}$ in LDL within a time that is a factor of 50 to 100 times longer than for HDL. Because liquid-like equilibrium was established in HDL at $205 \mathrm{~K}$ within several nanoseconds, we can access equilibrium LDL within a few hundred nanoseconds. On this basis, the distinct highand low-density phases observed on a submicrosecond time scale (Fig. 2) can be interpreted as quasi-equilibrated liquid phases. There is no immediate conversion to ice upon heating the sample, nor a continuous liquid-state conver- sion, as would be expected from the scenarios depicted in Fig. 1, C to E. The formation of crystalline ice would occur on time scales more than one order of magnitude longer than the conversion to LDL. It follows that our experimental data can only be quantitatively fit with the scenario shown in Fig. 1F (35), which shows the same discontinuous behavior in the x-ray scattering intensity as in the LLT of phosphorus (23).

To better characterize the progression of the LLT, we used scattering differences from the 35- to $55-\mu \mathrm{m}$-thick samples to estimate the fractional population of each phase in the sample as a function of the time delay (Fig. 4A) (35). We observed a small fraction of LDL at $16.8 \mathrm{~ns}$ that reached a maximum of $\sim 40 \%$ of the total scattering intensity at $3 \mu \mathrm{s}$ that was accompanied by a corresponding decrease in the HDL fraction. At $3 \mu \mathrm{s}$, crystalline ice appeared and became dominant at later times. The shape of the LDL scattering peak was not consistent with any substantial contributions of small nanocrystals of $\mathrm{I}_{\mathrm{sd}}$ when LDL first appeared (35). The formation of crystalline ice occurred on a time scale more than one order of magnitude longer than the conversion of HDL to LDL, demonstrating that the LLT, although a metastable phase transition, was a distinct process from the liquidto-ice transition.

Because of the dynamic nature of the decompression process, we expected that the conversion of HDL to LDL occurred in the region of the phase diagram between the equilibrium HDL-LDL coexistence line and the metastability limit (or spinodal) of the HDL phase. In

A
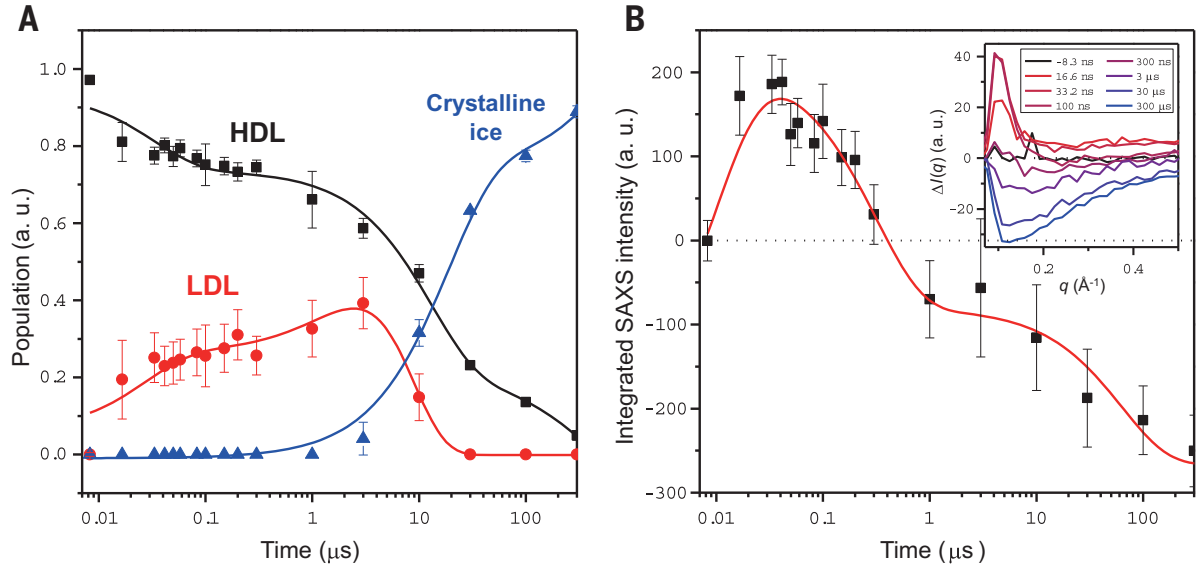

Fig. 4. Time-dependent population changes and SAXS intensity. (A) Time-dependent population changes of HDL (black squares), LDL (red circles), and crystalline ice (blue triangles). The solid black, red, and blue lines are shown to guide the eye. The error bar at each data point indicates the standard error determined from 20 independent measurements. (B) Time-dependent integrated SAXS intensity from $q=0.1$ to $0.3 \AA^{-1}$. The solid red line is shown to guide the eye. (Inset) The difference between the unpumped and pumped scattering curves at various time delays in the SAXS region. After $1 \mu \mathrm{S}$, the samples became more homogeneous than the unpumped sample, resulting in a negative SAXS difference. this region, the transition should manifest itself at short times as localized LDL fluctuations, followed by nucleation and growth of LDL domains as the decompression proceeds (47). Small LDL fluctuations were evident in Fig. 4B, which shows the small-angle $\mathrm{x}$-ray cattering (SAXS) intensity in the range from 0.1 to $0.3 \AA^{-1}$ as a function of the time delay. There was a SAXS enhancement at short times peaking between 50 and $100 \mathrm{~ns}$. The estimated correlation length, obtained by fitting the SAXS curves, peaks at 10 to $20 \AA$ in the same time interval (35).

Lluctuations of this size appearing with in the HDL phase would result in some contribution of scattering between atom pairs being in both LDL and HDL and would cause fect the LDL peak position in $q$-space. Such interference has been observed in the converon unannealed HDA (UHDA), in contrast to expanded HDA (eHDA) (18). At early time delays, we observed such a shift (35), indicating that interference did arise from small SAXS information.

At time delays longer than $100 \mathrm{~ns}$, the interference was almost gone, and the SAXS enhancement was no longer visible, suggesting that the LDL domains grew to macroscopic size, and the contribution of atom pairs across interface became negligible. Although the complete conversion of the sample to LDL was preempted by ice crystallization, for our thin samples, the HDL:LDL ratio reached 1:1 at $1 \mu \mathrm{s}$ before any ice appeared (Fig. 2B). Nucleation and growth of LDL domains within HDL was

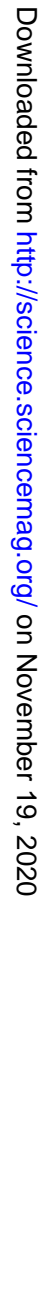


only consistent with a discontinuous firstorder LLT and would not be observed in a continuous transformation of a spatially homogenous system from high to low density.

Our results reveal structural changes in liquid water at $205 \mathrm{~K}$ consistent with crossing a discontinuous LLT between HDL and LDL phases occurring at a pressure between ambient and $3.5 \mathrm{kbar}$. Although some uncertainty exists in our estimate of the temperature (195 to $215 \mathrm{~K}$ ) at which the decompression process occurs in our experiments, our results show that an LLT occurred in the high-temperature region of the "no-man's land" (pressures and temperatures at which usually only crystallization occurs), and well above the temperature range of the amorphous ice phases, as confirmed by partially melting of hexagonal ice by using our IR heating procedure. Furthermore, the time scale we observed for the LLT (nanoseconds to microseconds) is consistent with a previous experimental estimate, based on extrapolations from $10 \mathrm{~ms}$ at $174 \mathrm{~K}$ to nanoseconds and microseconds at $220 \mathrm{~K}$ by using temperature-dependent kinetic measurements analyzed with an Arrhenius expression (42).

It has been observed that at 1 bar, crystallization occurs on a 10- to $100-\mu$ s time scale at $\sim 8 \mathrm{~K}$ below $T_{\mathrm{H}}(8)$. Considering that we observed that ice crystallization from the LDL phase occurred on the same time scale, we expect that the HDL spinodal of the LLT (at $\approx 205 \mathrm{~K}$ ) is a few degrees below $T_{\mathrm{H}}$ and follows closely the homogeneous ice nucleation line (43). A more narrow range of 1.5 to $2 \mathrm{kbar}$ for the HDL spinodal is consistent with a recent LLT study that used a waterrich ideal solution that prevented crystallization (48).

Our observation of an LLT during isothermal decompression at positive pressure, combined with water at 1 bar changing continuously on cooling, implies the existence of an LLCP at positive pressures $(7,8)$. We expect that the procedure presented here could provide more details about the nature of the LLT and help refine the location of the LLCP. The latter could be identified from critical fluctuations observed through SAXS intensity enhance- ments at lower $q$ than in the present study, thus allowing detection of a diverging correlation length (6).

\section{REFERENCES AND NOTES}

1. R. J. Speedy, C. A. Angell, J. Chem. Phys. 65, 851-858 (1976)

2. C. A. Angell, W. J. Sichina, M. Oguni, J. Phys. Chem. 86 998-1002 (1982)

3. P. H. Poole, F. Sciortino, U. Essmann, H. E. Stanley, Nature 360, 324-328 (1992).

4. S. Sastry, P. G. Debenedetti, F. Sciortino, H. E. Stanley, Phys. Rev. E Stat. Phys. Plasmas Fluids Relat. Interdiscip. Topics 53 6144-6154 (1996).

5. R. J. Speedy, J. Phys. Chem. 86, 982-991 (1982).

6. P. G. Debenedetti, F. Sciortino, G. H. Zerze, Science 369 , 289-292 (2020).

7. K. H. Kim et al., Science 358, 1589-1593 (2017).

8. J. A. Sellberg et al., Nature 510, 381-384 (2014)

9. T. A. Kesselring, G. Franzese, S. V. Buldyrev, H. J. Herrmann, H. E. Stanley, Sci. Rep. 2, 474 (2012).

10. D. T. Limmer, D. Chandler, J. Chem. Phys. 138, 214504 (2013)

11. E. B. Moore, V. Molinero, Nature 479, 506-508 (2011).

12. J. C. Palmer et al., Nature 510, 385-388 (2014).

13. F. Smallenburg, F. Sciortino, Phys. Rev. Lett. 115, 015701 (2015).

14. S. D. Overduin, G. N. Patey, J. Chem. Phys. 138, 184502 (2013)

15. R. S. Singh, J. W. Biddle, P. G. Debenedetti, M. A. Anisimov, J. Chem. Phys. 144, 144504 (2016).

16. J. C. Palmer et al., J. Chem. Phys. 148, 137101 (2018)

17. O. Mishima, Y. Suzuki, Nature 419, 599-603 (2002).

18. F. Perakis et al., Proc. Natl. Acad. Sci. U.S.A. 114, 8193-8198 (2017).

19. K. Winkel, E. Mayer, T. Loerting, J. Phys. Chem. B 115 14141-14148 (2011)

20. C. A. Tulk, J. J. Molaison, A. R. Makhluf, C. E. Manning, D. D. Klug, Nature 569, 542-545 (2019).

21. J. S. Tse et al., Nature 400, 647-649 (1999).

22. J. J. Shephard, C. G. Salzmann, J. Phys. Chem. Lett. 7 , 2281-2285 (2016).

23. Y. Katayama et al., Nature 403, 170-173 (2000).

24. D. S. Yang, A. H. Zewail, Proc. Natl. Acad. Sci. U.S.A. 106, 4122-4126 (2009).

25. A. K. Soper, M. A. Ricci, Phys. Rev. Lett. 84, 2881-2884 (2000).

26. J. L. Finney, A. Hallbrucker, I. Kohl, A. K. Soper, D. T. Bowron, Phys. Rev. Lett. 88, 225503 (2002).

27. D. Mariedahl et al., J. Phys. Chem. B 122, 7616-7624 (2018).

28. Y. Xu, N. G. Petrik, R. S. Smith, B. D. Kay, G. A. Kimmel, Proc. Natl. Acad. Sci. U.S.A. 113, 14921-14925 (2016).

29. S. Woutersen, B. Ensing, M. Hilbers, Z. Zhao, C. A. Angell, Science 359, 1127-1131 (2018).

30. K. S. Kjær et al., Phys. Chem. Chem. Phys. 15, 15003-15016 (2013)

31. K. Ramasesha, L. De Marco, A. Mandal, A. Tokmakoff, Nat. Chem. 5, 935-940 (2013).

32. H. Iglev, M. Schmeisser, K. Simeonidis, A. Thaller, A. Laubereau, Nature 439, 183-186 (2006).

33. N. Linz, S. Freidank, X. X. Liang, A. Vogel, Phys. Rev. B 94 024113 (2016)

34. J. Savolainen, F. Uhlig, S. Ahmed, P. Hamm, P. Jungwirth, Nat. Chem. 6, 697-701 (2014)
35. Materials and methods are available as supplementary materials.

36. S. Fanetti et al., J. Phys. Chem. Lett. 10, 4517-4522 (2019).

37. K. Amann-Winkel et al., Proc. Natl. Acad. Sci. U.S.A. 110, 17720-17725 (2013)

38. O. Mishima, J. Chem. Phys. 133, 144503 (2010).

39. V. Holten, M. A. Anisimov, Sci. Rep. 2. 713 (2012)

40. L. B. Skinner et al., J. Chem. Phys. 144, 134504 (2016).

41. L. B. Skinner, C. J. Benmore, J. C. Neuefeind, J. B. Parise, J. Chem. Phys. 141, 214507 (2014).

42. C. Lin, J. S. Smith, X. Liu, J. S. Tse, W. Yang, Phys. Rev. Lett. 121, 225703 (2018).

43. H. Kanno, R. J. Speedy, C. A. Angell, Science 189, 880-881 (1975).

44. P. G. Debenedetti, F. H. Stillinger, Nature 410, 259-267 (2001).

45. F. X. Prielmeier, E. W. Lang, R. J. Speedy, H. D. Lüdemann, Ber. Bunsenges. Phys. Chem 92, 1111-1117 (1988).

46. C. Lin, J. S. Smith, S. V. Sinogeikin, G. Shen, Proc. Natl. Acad. Sci. U.S.A. 115, 2010-2015 (2018).

47. K. Takae, H. Tanaka, Proc. Natl. Acad. Sci. U.S.A. 117 4471-4479 (2020)

48. Z. Zhao, C. A. Angell, Angew. Chem. Int. Ed. 55, 2474-2477 (2016)

\section{ACKNOWLEDGMENTS}

Funding: This work has been supported by a European Research Council Advanced Grant under project 667205 and the Swedish National Research Council. K.A.-W. acknowledges funding by the Ragnar Söderbergs Stiftelse. T.J.L. was supported by by U.S. Department of Energy contract DE-AC02-76SF00515. This work is also supported by the National Research Foundation of Korea (NRF) grant funded by the Korea government (MSIT) (2019R1C1C1006643). The experiments were performed at beamline XSS of PAL-XFEL (proposals 2018-1st-XSS-009, 20182nd-XSS-006, and 2019-1st-XSS-008) funded by the Korea government (MSIT). P.H.P. is supported by the Natural Sciences and Engineering and Research Council (Canada). The simulations were supported by ACENET and Compute Canada. Author contributions: A.N. designed and supervised the study. K.A.-W. designed sample preparation. K.A.-W and A.S designed sample holder. K.A.-W., D.M., T.E., A.S., and M.L.P. prepared ice samples. K.H.K., K.A.-W., A.N., A.S., F.P., H.P., and M. W. designed experimental setup, chamber, and laser geometry. K.H.K., K.A.-W., A.N., A.S., F.P., H.P., M.L.P., C.Y., D.M., T.E., T.J.L, S.Y., S.J., J.H.L., I.E., M.K., J.P., and S.H.C. performed the X-ray experiments. N.G. and P.H.P. performed the computer simulations. K.H.K., A.S., C.Y., S.Y., and S.J. analyzed the data. A.N., K.H.K., K.A.-W., A.S., H.P., M.L.P., P.H.P., N.G., and T.J.L. wrote the manuscript. Competing interests: The authors declare no competing interests. Data materials availability: All data necessary for evaluating the conclusions of the study are included in the supplementary materials.

\section{SUPPLEMENTARY MATERIALS}

science.sciencemag.org/content/370/6519/978/suppl/DC1 Materials and Methods

Supplementary Text

Figs. S1 to S17

Tables $\mathrm{S} 1$ and $\mathrm{S} 2$

References (49-79)

27 March 2020; resubmitted 11 August 2020

Accepted 6 October 2020

$10.1126 /$ science abb9385 


\section{Science}

\section{Experimental observation of the liquid-liquid transition in bulk supercooled water under pressure}

Kyung Hwan Kim, Katrin Amann-Winkel, Nicolas Giovambattista, Alexander Späh, Fivos Perakis, Harshad Pathak, Marjorie Ladd Parada, Cheolhee Yang, Daniel Mariedahl, Tobias Eklund, Thomas. J. Lane, Seonju You, Sangmin Jeong, Matthew Weston, Jae Hyuk Lee, Intae Eom, Minseok Kim, Jaeku Park, Sae Hwan Chun, Peter H. Poole and Anders Nilsson

Science 370 (6519), 978-982

DOI: $10.1126 /$ science.abb9385

\section{Liquid-liquid transitions under pressure}

Theoretical simulations suggest that deeply supercooled water undergoes a transition between high- and low-density forms, but this transition is difficult to study experimentally because it occurs under conditions in which ice crystallization is extremely rapid. Kim et al. combined x-ray lasers for rapid structure determination with infrared femtosecond pulses for rapid heating of amorphous ice layers formed at about 200 kelvin. The heating process created high-density liquid water at increased pressures. As the layer expanded and decompressed, low-density liquid domains appeared and grew on time scales between 20 nanoseconds and 3 microseconds, which was much faster than competing ice crystallization.

Science, this issue p. 978

ARTICLE TOOLS

SUPPLEMENTARY

MATERIALS

REFERENCES

PERMISSIONS http://science.sciencemag.org/content/370/6519/978

http://science.sciencemag.org/content/suppl/2020/11/18/370.6519.978.DC1

This article cites 79 articles, 11 of which you can access for free http://science.sciencemag.org/content/370/6519/978\#BIBL

http://www.sciencemag.org/help/reprints-and-permissions

Use of this article is subject to the Terms of Service

Science (print ISSN 0036-8075; online ISSN 1095-9203) is published by the American Association for the Advancement of Science, 1200 New York Avenue NW, Washington, DC 20005. The title Science is a registered trademark of AAAS.

Copyright (C) 2020 The Authors, some rights reserved; exclusive licensee American Association for the Advancement of Science. No claim to original U.S. Government Works 